Authors' version, the final publication is available at: https://link.springer.com/chapter/10.1007/978-3-319-55961-2_27

\title{
A case study on Linked Data for University Courses
}

\author{
Barnabás Szász ${ }^{1}$, Rita Fleiner², András Micsik ${ }^{3}$ \\ ${ }^{1}$ University of Debrecen, Debrecen, Hungary \\ bszasz@gmail.com \\ ${ }^{2}$ Óbuda University, Budapest, Hungary \\ fleiner.rita@nik.uni-obuda.hu \\ ${ }^{3}$ MTA SZTAKI, Budapest, Hungary \\ micsik@sztaki.mta.hu
}

\begin{abstract}
University wanted to build a linked dataset describing their courses in the semester. The concepts to be covered included curricula, subjects, courses, semesters and educators. A particular use case needed the description of lecture rooms and events as well. Although there are several ontologies for the mentioned domains, selecting a set of ontologies fitting our use case was not an easy task. After realizing the problems, we created the Ontology for Linked Open University Data (OLOUD) to fill in the gaps between re-used ontologies. OLOUD acts as a glue for a selection of existing ontologies, and thus enables us to integrate data from several sources and to provide practical information services for students and lecturers.
\end{abstract}

Keywords: Ontology $\cdot$ Linked Open Data $\cdot$ Linked Open University Data $\cdot$ SPARQL

\section{$1 \quad$ Introduction}

In this paper we focus on a special segment of open data at the university domain: university courses. We aim to facilitate the implementation of Smart Universities [1] by defining a common data model for course information. Ontological representation as the most modern description method for the problem domain was chosen. Originally our objective was to develop a generic data model for university course related data. During our work we noticed that though the Bologna Process ensures a certain level of compatibility for education systems in the EU, this does not reach deeper constructs regarding the educational model. We found that the meaning of the main concepts (like course, subject and study programme) is quite different in currently available educational models in Europe.

Presenting course related information requires a lot of data originating from multiple information systems at a typical university. As these systems are usually not fully integrated and the access to the data is limited, significant effort is necessary to successfully navigate through the potential difficulties. Foreign students, who are not aware of the local specialties can find it even more cumbersome. With our data model 
we would like to support the generation and the management of integrated university data and also the appearance of future mobile and web applications building on the use of this data.

In Section 2 use cases are explored for the planned course dataset. Section 3 is about existing work related to our goals. Section 4 clarifies the terminology and describes how our new "glue" ontology was built and how it re-uses other existing ontologies. Finally, we summarize results in Section 5.

\section{Use Case}

In the following we list some of the tasks we aim to support with our Linked Data approach.

Courses. Courses are organized into a series of lectures and lab exercises, either in a weekly or in a custom cadence within a semester. There might be multiple labs advertised for a course, so students can choose the most suitable to their circumstances. This creates the challenge of assembling a personal timetable for students and lecturers avoiding conflicts and considering personal preferences and requirements.

Students could benefit from an integrated view containing course description (title, identifier, abstract, dependencies), course time, location, and learning resources. A personal information service may provide students with on-demand information about their daily schedule, navigation to the next lecture, overlaps of classes, etc. We also consider accessible way-finding to course locations.

Curriculum fulfillment. There is a need for long term planning of studies as well. Quite often there are no predefined course timetables at Hungarian universities, just a list of courses to be completed, and a dependency graph among the courses, which defines the prerequisites for each. Some courses are advertised in every second semester only. Some universities recommend a specific order of courses, but following such an order breaks easily if for example a single course is not completed in the suggested semester. Thus, students face a kind of constraint satisfaction problem to solve at each semester start.

For this purpose, students need a personal advisor recommending the best way for them to fulfill the curriculum requirements. This advisor needs to consider where the student is on his roadmap, what courses they should focus on, what are the personal preferences (e.g. preferred number of courses or credits per semester) and what courses are being advertised.

Resource reservation. University resources (rooms, equipment) are used by multiple faculties. They can be booked for regular courses, exams in the exam period and other events. Different types of events may have separate registries, thus blocking an overall view of anticipated resource usages. One needs an overall list of reservations by reserving person, location and date at least. 


\section{$3 \quad$ Related Work}

Linked Universities $^{1}$ and Linked Education ${ }^{2}$ are two European initiatives created to enable education with the power of Linked Data. Linked Universities is an alliance of European universities engaged into exposing their public data as linked data. LinkedEducation.org is an open platform aimed at further promoting the use of Linked Data for educational purposes. However, we did not find any existing solution or vocabulary at these websites fulfilling our needs for the data model.

The Open University in the UK was the first university that created a linked data platform to expose information from its departments [2]. The Open University datasets can be classified in the following six groups: open educational resources, scientific production, social media, organizational data, research project output, publication metadata. The main difference with Óbuda University is the lack of navigation and timetable data at the Open University.

The general process for building linked open university data and a use case at Tsinghua University are described in [3]. Procedures like choosing datasets and vocabularies, collecting and processing data, converting data into RDF and interlinking datasets are studied. The datasets unfortunately are not available through public SPARQL endpoint.

The Lucero project analyzed open educational datasets in 2012 [4]. Linked Open Datasets in four universities and four broader educational projects were studied and the most commonly used vocabularies, classes and properties were described. In this case no representations for course, semester or lecture room concepts were found. The general state of linked data for education is studied in [5] containing statistics on vocabulary re-use. We found another very useful review of ontologies for modelling course information in higher education [6].

AIISO (Academic Institution Internal Structure Ontology) [7] provides classes and properties to describe the structure of an academic institution. It is designed to be used in conjunction with the Participation ontology [8] which stands for describing the roles that people play within groups.

TEACH [9] is a lightweight vocabulary providing detailed properties in order to describe a course, but it doesn't model the provider of the course. The concepts in TEACH lack some important features that are essential for our purposes. For example, in order to describe university courses the concept of Subject is necessary, which doesn't exist in TEACH.

XCRI-CAP [10] is the abbreviation for eXchanging Course Related Information, Course Advertising Profile. It is the UK standard for describing course marketing information. XRI-CAP doesn't make a distinction between a module, a course, a subject or a study program.

The Metadata for Learning Opportunities (MLO) Advertising ontology [11] aims to standardize the specifications for describing and exchanging information about courses and learning opportunities. It can be considered the European equivalent of the British Standard XCRI-CAP for advertising courses. MLO-Adv contains very abstract and general concepts and misses many properties for courses and curricula.

\footnotetext{
${ }^{1}$ http://linkeduniversities.org

${ }^{2} \mathrm{http}$ ://linkededucation.org
} 
After reviewing the above ontologies, we realized that there are many ontologies defining Course, Subject, Curriculum and Degree, but neither provides a full coverage and there are missing relations between the concepts. We concluded that the AIISO ontology provides the best coverage, and its structure fits our concrete use case, thus it can be reused and extended with the necessary terms.

\section{$4 \quad$ Implementation}

The major concepts for university students and teachers in our use case are depicted in Figure 1.

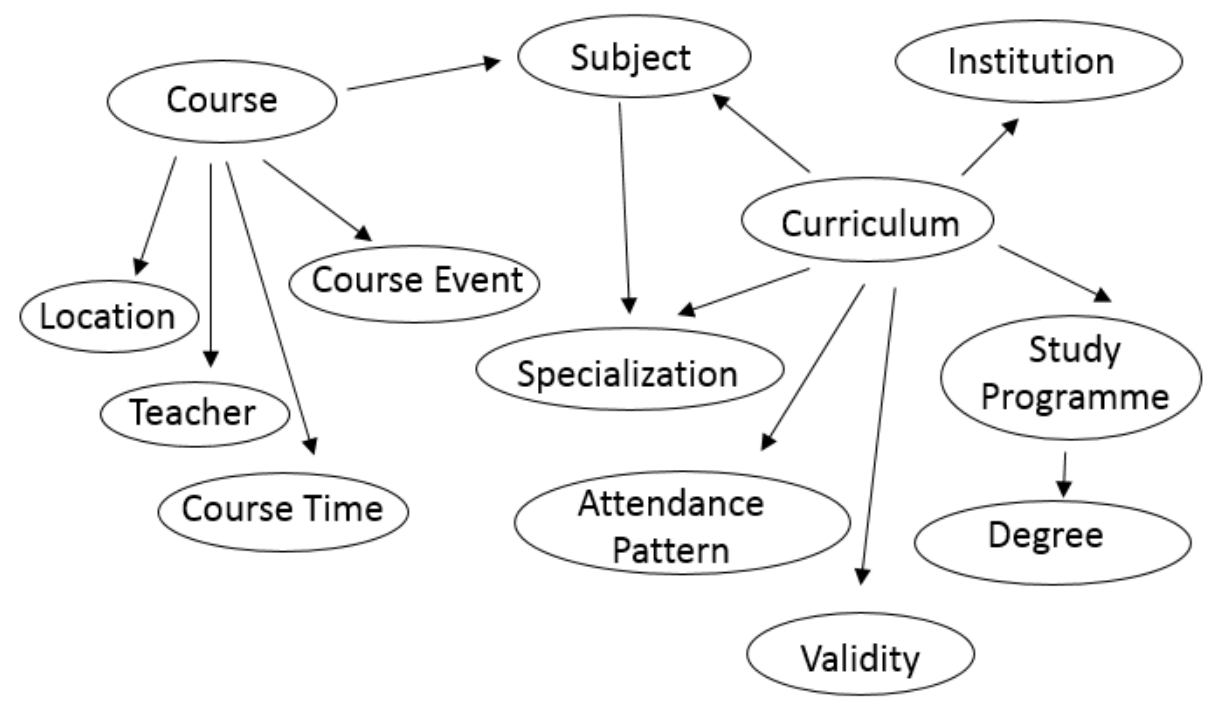

Fig. 1. Main concepts for our use cases

A Curriculum stands in many-to-one relationship with a Study Programme (e.g. Applied Mathematician), offered by the university. Each Curriculum has a specific Attendance Pattern (full-time, part-time, correspondence, etc.). A Study Programme determines the qualification and a specific Degree (BSc, MSc, BA, MA, PhD, etc.) that students will get after the successful completion of their studies. A Study Programme must be accredited by an external body. The curriculum is the specification how the Study Programme can be completed. A Curriculum is valid for a given time interval, meaning that a student can be assigned to it only if his enrollment time falls into this period of time. For each Subject there is an Organizational Unit responsible for it. Courses are advertised based on a Subject, have temporal (Course Time) and spatial (Location) attributes and one or more assigned Teacher(s). Course Events are individual sessions such as lectures or practice held often on a weekly basis, and courses may also have some special, associated events, for example exams. Addition- 
ally, learning material (Learning Resource) can be assigned to specific courses and subjects.

Course is the elementary unit of the educational process, where students, teachers, location and date are assigned. Subject is a higher level component of the training process, it represents a part of knowledge students need to acquire.

As seen in Section 3, we had to extend and connect existing ontologies for our domain model. The result is the Ontology for Linked Open University Data (OLOUD) presented in the rest of the section. Figure 2 depicts the main classes as ovals and the most significant attributes as arrows in OLOUD.

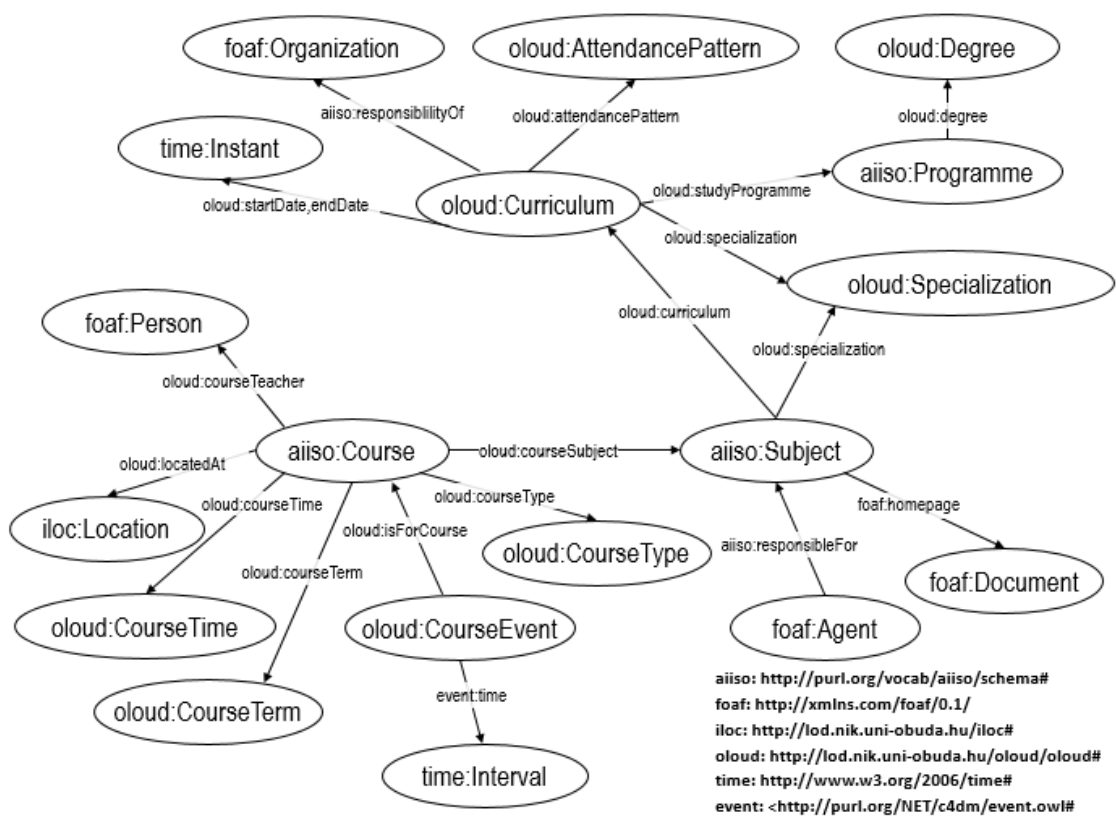

Fig. 2. Main classes and properties in OLOUD

The prefixes for classes and properties clearly show what has been reused and where we had to fill missing links. We selected to reuse the following ontologies: AIISO for the description of university faculties, courses, subjects and roles; FOAF for the description of persons; OWL Time for temporal definitions; Event for describing events and Dublin Core for generic properties such as language.

Curriculum, Subject, Course and CourseEvent are the most important classes in OLOUD. In the following the description of the classes are given with their direct connections. Curricula contain the list of subjects with their dependencies, since each subject can have various prerequisite subjects. The faculty of the university responsible for the Curriculum is given by the aiiso:responsibility of property. StartDate and endDate properties determine the period, when a Curriculum is valid. The possible Specializations, the Degree, the Attendance pattern and the Study programme of the study are given by the corresponding specialization, degree, attendancePattern and 
studyProgramme properties. The language of the studies is given with the dcterms:language property.

Subjects are described by their name, code, credit number, person and organization responsible for it: foaf:name, aiiso:code, subjectCredit, aiiso:responsibilityOf. The connection between a Subject and its Courses is given by the courseSubject attribute. The prerequisite conditions between Subject entities are set by the hasPrerequisite property.

The individuals of the Course class are the actual instances of subjects running in a given semester (courseTerm) having spatial, temporal and type descriptions, identification number, name and instructor: locatedAt, courseTime, courseTerm, courseType, aiiso: code, foaf:name and courseTeacher.

CourseEvent class represents the individual classes or exams associated to a Course instance. Its temporal description is given by the event:Interval class, and the connection between a course event and its time is given by the event:time property. The isForCourse property is used to connect a CourseEvent with a Course.

New classes are introduced for the full description of the Curriculum, Subject and Course individuals including StudyProgramme, Degree, AttendancePattern, Specialization, CourseTerm and CourseType.

Entities describing indoor locations for Course and Event individuals are represented by the Location class from the $\mathrm{iLOC}^{3}$ ontology. Courses and events can be assigned to Rooms, and Rooms are connected via a network of POIs (Points of Interest), which can be doors, hallway connections, etc. The offices of lecturers can also be included in the description of campus buildings.

OWL Time [12] and the TimeAggregates Ontology [13] are used to express temporal descriptions of courses. Our objective was to enable SPARQL queries according to date, time and duration and to define course time as recurring events (e.g. labs on every Tuesday from 9 am till 10.30 am in the 2016 Fall semester). The triples representing such information need several additional individuals, hence the management of such information is time consuming and error prone. The generation of course time data was implemented using an automatized process described in [14] and complete examples can be found on the web ${ }^{4}$.

The advantage of OLOUD as a common vocabulary is the ability to integrate databases covering only parts of university life. Then, the unified data can be queried using SPARQL to find answers for complex queries relating multiple aspects such as timetable, location, curriculum, etc. simultaneously. The next part of our research is to further investigations on OLOUD as a common model, and to discover constraints and rules for university domain, resulting in a unified knowledge base which can be used for inferencing new facts and for finding inconsistencies, problems in campus data. Reasoners may reveal problems in current data such as course overlaps, missing courses for subjects, or cycles in subject prerequisites (see following example rules).

hasPrerequisite(?x, ?y), hasPrerequisite(?y, ?z) -> hasPrerequisite(?x, ?z)

Subject(?x), hasPrerequisite(?x, ?x) -> SelfDependentSubject(?x)

Finally, rules run on temporary facts can be used to generate personal short or longterm tuition plans for students.

\footnotetext{
${ }^{3}$ http://lod.nik.uni-obuda.hu/iloc/iloc.html

${ }^{4}$ http://lod.nik.uni-obuda.hu/unfolding/example.html
} 


\section{$5 \quad$ Results}

Protégé ${ }^{5}$ was used for validation purposes, as it revealed problems in the imported ontologies and in our ontology as well. The OOPS! Ontology Pitfall Scanner was also used to check our OWL [15], and the found small problems were fixed.

Linked data triplets were created based on public data ${ }^{6}$ at the Obuda University. The location data was created manually based on building layout diagrams of the university, while the subject and course data were converted using custom PHP scripts from relational database dumps extracted from the electronic administration system (Neptun) of the Óbuda University. The entities of the Person class and their personal data were scraped from the personal webpages. The university event descriptions were generated with a crawler from the OU webpage. The dataset was also extended with links to the GeoNames geographic dataset. At this moment, the database contains about 1000 entities with more than 6000 triples.

As an example, we provide a sample Course, Subject and Curriculum description:

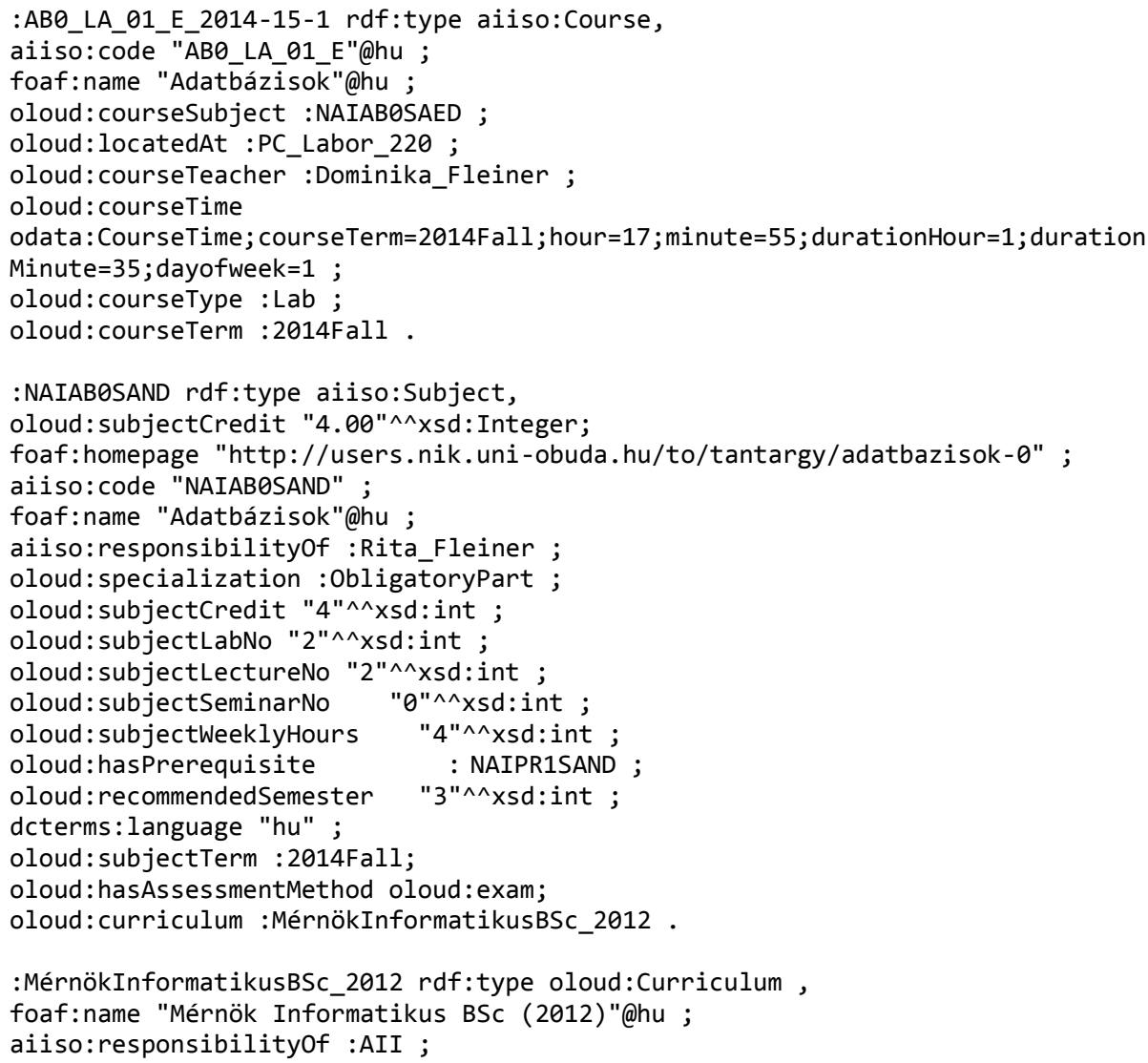

${ }^{5} \mathrm{http}: / /$ protege.stanford.edu/

${ }^{6}$ http://lod.nik.uni-obuda.hu/marmotta/ 
oloud:studyProgramme :ComputerEngineer ;

oloud:attendancePattern :FullTime ;

dcterms:language "hu" ;

oloud:degree :bsc ;

oloud: startDate odata: UnitDayInstant; year=2012; month=9; day=1.

Figure 3 provides a visualization of a part of the linked data graph. Browsing the dataset with a LOD tool such as LODmilla [16] provides access to useful integrated data for students. Furthermore, an in-door way finding service for students is also available, providing a simple itinerary to the place of the given lecture. It is available as a mobile app, and backed by an open SPARQL endpoint.

We tested the complex use cases with implementing different SPARQL queries answering the questions in Section 2. For example, one can ask about the courses for a given subject in a specific semester:

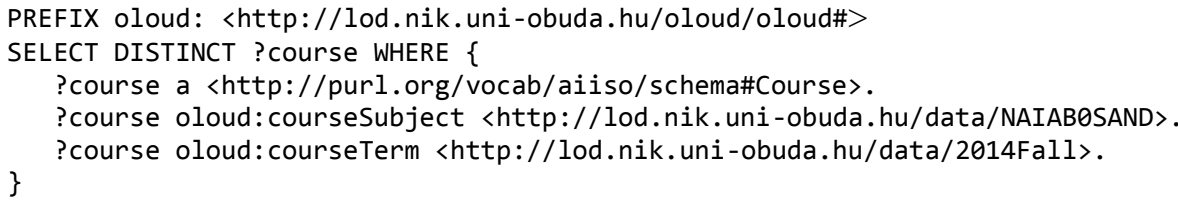

What is the course schedule (with course identifier, time and lecturer) for a specific lecture hall or lab?

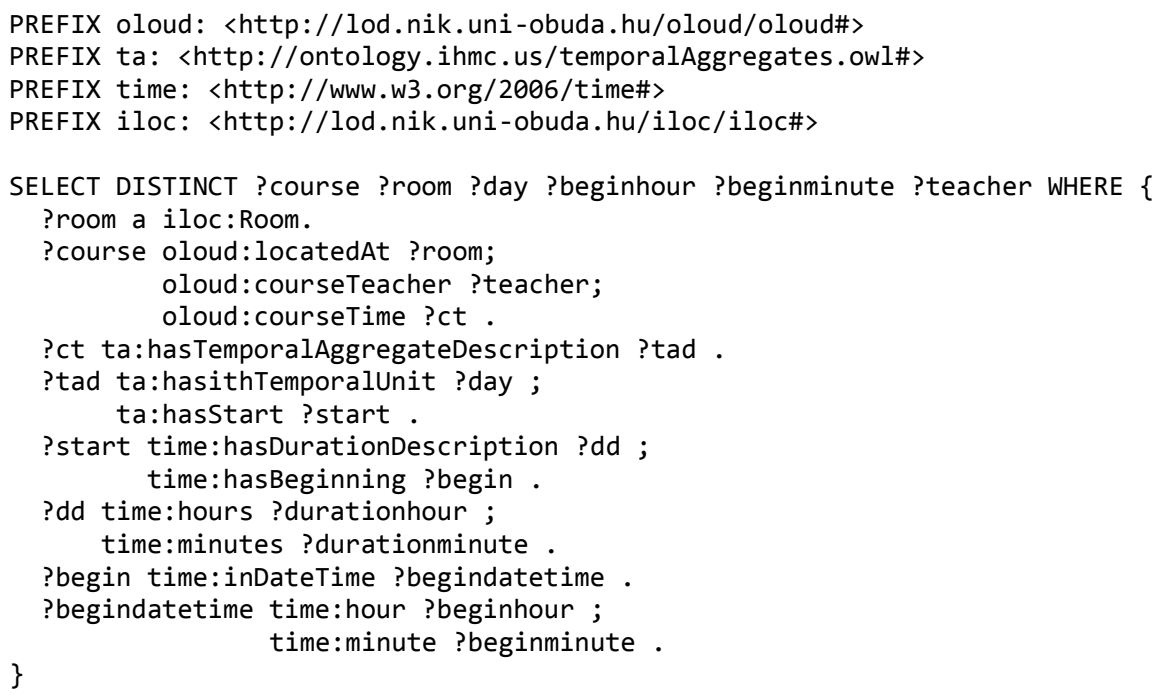

What are the prerequisite subjects of a specific subject in the curriculum?

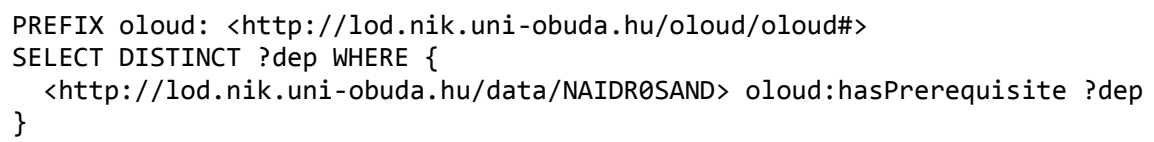


Further sample SPARQL queries and more information about the dataset and the ontology can be found on the ontology homepage ${ }^{7}$.

On our LOD server we currently serve the dataset using Marmotta ${ }^{8}$, but we also experiment with Virtuoso. In the future we wish to proceed with various application developments using the generated LOD dataset. For example, a 'curriculum assistant' mobile application helping students to select their courses at the start of the semester might be useful.

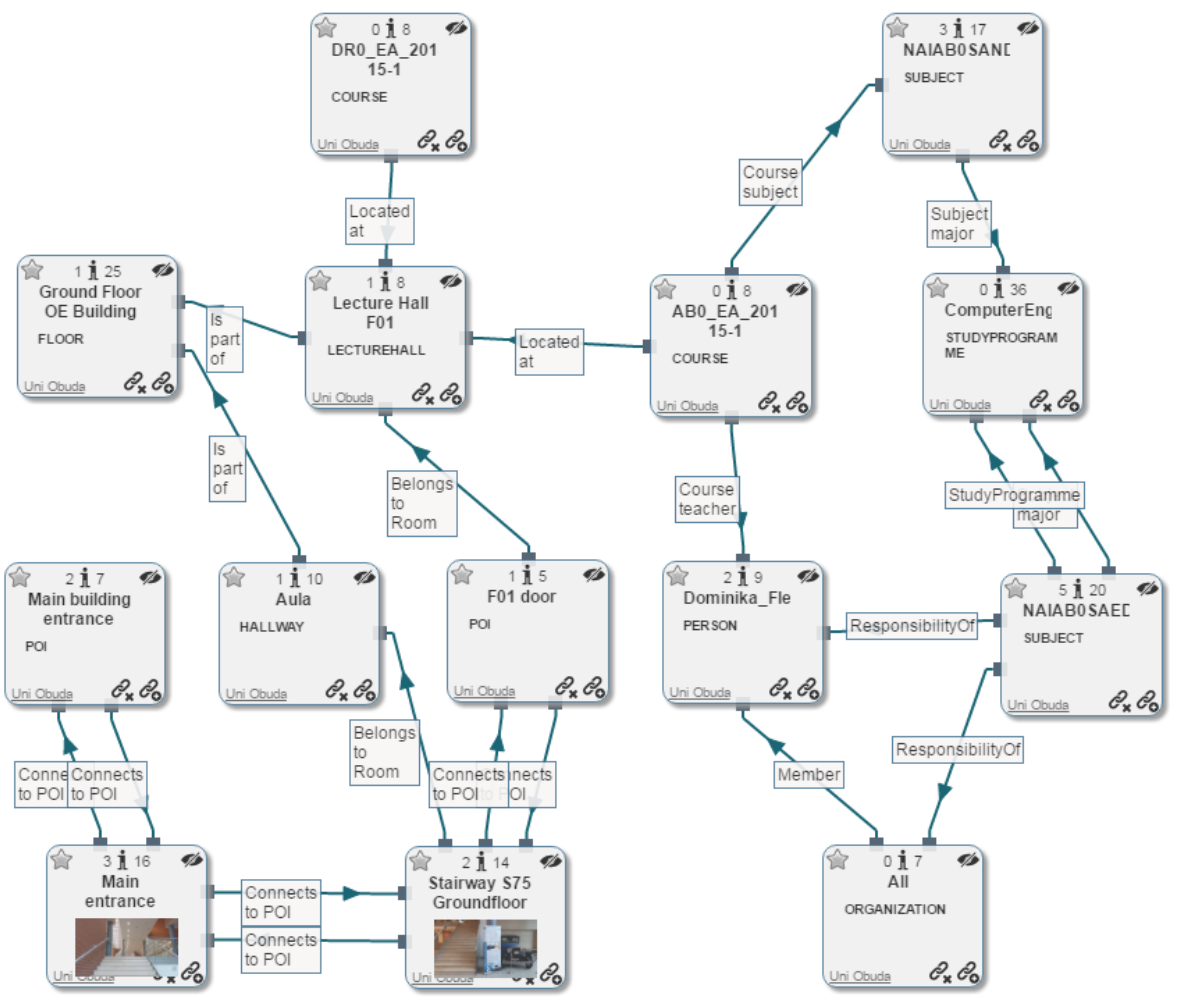

Fig. 3. Extract of the dataset presented with the Lodmilla browser

\section{Conclusions}

With an initial goal to implement useful, "smart" services for university students based on linked data, we soon realized that there are many ontologies or vocabularies for the domain, yet none of them is suitable for our purpose. The biggest problems we found were the missing discriminations between subjects and courses and curricula and study programs. We created the OLOUD ontology which amalgamates selected ontologies and facilitates the full description of course-related information. Hence, we

\footnotetext{
${ }^{7}$ http://lod.nik.uni-obuda.hu/oloud/

${ }^{8} \mathrm{http}: / /$ marmotta.apache.org/
} 
think our work helps to better clarify the role of concepts frequently used in this domain.

The OLOUD ontology provides the basis of several ongoing student projects, which either integrate new datasets for the university or implement new services on top of the OLOUD dataset. Furthermore, OLOUD aims to become more than a vocabulary and to exploit the modeling power of description logic by exploring and describing the constraints and details of concepts in this domain.

\section{References}

1. Rohs, M., \& Bohn, J.: Entry points into a smart campus environment-overview of the ETHOC system. In 23rd International Conference on Distributed Computing Systems, Workshops Proceedings, pp. 260-266, IEEE (2003)

2. Daga, E., d'Aquin, M., Adamou, A., Brown, S.: The Open University Linked Data - data.open.ac.uk. Semantic Web Journal, 7(2) pp. 183-191. (2015)

3. Ma, Y., Xu, B., Bai, Y., Li, Z.: Building linked open university data: Tsinghua university open data as a showcase. In: The Semantic Web, pp. 385-393., Springer Berlin Heidelberg (2011)

4. The Lucero project: So, what's in linked datasets for education (2012) http://lucero-project.info/lb/2012/04/so-whats-in-linked-datasets-for-education/

5. d'Aquin, M., Adamou, A., Dietze, S.: Assessing the educational linked data landscape. In: Proceedings of the 5th Annual ACM Web Science Conference, pp. 43-46. ACM (2013)

6. Barker, P.: A short project on linking course data (2015) http://blogs.pjjk.net/phil/a-short-project-on-linking-course-data/

7. Styles, R., Shabir, N.: Academic institution internal structure ontology (aiiso). (2008) http://vocab.org/aiiso/schema

8. Styles, R., Wallace, C., Moeller, K.: Participation ontology. (2008) http://vocab.org/participation/schema

9. Kauppinen, T., Trame, J., Westermann, A.: Teaching Core Vocabulary Specification (TEACH ontology). (2012) http://linkedscience.org/teach/ns/

10. Stubbs, M., Wilson, S.: eXchanging Course-Related Information: a UK service-oriented approach. (2006) http://hdl.handle.net/1820/837

11. EN 15982:2011 Metadata for Learning Opportunities (MLO)-Advertising.

12. OWL Time Ontology. https://www.w3.org/TR/owl-time/

13. Pan, F.: Temporal aggregates for Web services on the semantic Web. IEEE International Conference on Web Services (ICWS'05), pp. 831-832. IEEE (2005)

14. Szasz, B., Fleiner, R., Micsik, A.: Linked data enrichment with self-unfolding URIs. In 2016 IEEE 14th International Symposium on Applied Machine Intelligence and Informatics (SAMI) pp. 305-309. IEEE (2016)

15. Poveda-Villalón, M., Suárez-Figueroa, M. C., \& Gómez- Pérez, A.: Validating ontologies with OOPS!. In Knowledge Engineering and Knowledge Management, pp. 267-281. Springer Berlin Heidelberg (2012)

16. Micsik, A., Turbucz, S., Györök, A.: LODmilla: a Linked Data Browser for All. In: Proceedings of the Posters and Demos Track of 10th International Conference on Semantic Systems - SEMANTiCS2014, pp. 31-34. CEUR Workshop Proceedings (2014) 